\title{
Typing of the pilus-protein-encoding FCT region and biofilm formation as novel parameters in epidemiological investigations of Streptococcus pyogenes isolates from various infection sites
}

\author{
Thomas Köller, ${ }^{1} \dagger$ Andrea Guido Oreste Manetti, ${ }^{\dagger} \dagger$ Bernd Kreikemeyer, ${ }^{1}$ \\ Cordula Lembke, ${ }^{1}$ Immaculada Margarit, ${ }^{2}$ Guido Grandi ${ }^{2}$ \\ and Andreas Podbielski ${ }^{1}$ \\ ${ }^{1}$ Institute of Medical Microbiology, Virology and Hygiene, University Hospital, Schillingallee 70, \\ D-18057 Rostock, Germany \\ ${ }^{2}$ Novartis Vaccines and Diagnostics, Via Fiorentina 1, 53100 Siena, Italy
}

Correspondence

Bernd Kreikemeyer

bernd.kreikemeyer@med.unirostock.de

Received 16 June 2009

Accepted 9 December 2009

\section{INTRODUCTION}

Streptococcus pyogenes [group A streptococci (GAS)] is a very common and predominantly human pathogen causing a wide variety of diseases. The most frequent diseases are mild suppurative throat and skin infections (estimated 727 million cases per year worldwide). Severe invasive diseases (estimated 663000 cases per year) like meningitis, necrotizing fasciitis and the streptococcal toxic shock syndrome

†These authors contributed equally to this paper.

Abbreviations: CLSI, Clinical and Laboratory Standards Institute; GAS, group A streptococci.

A table of isolate information and figures of FCT-typing and biofilmassay data are available as supplementary material with the online version of this paper. occur more rarely (Carapetis et al., 2005; Cunningham, 2000; Bisno, 1991; Bisno \& Stevens, 1996; Wahl et al., 2007; Stevens, 1996). S. pyogenes also causes non-suppurative post-infection sequelae (1.1 million cases per year) such as rheumatic fever, acute glomerulonephritis and obsessive compulsive neurological disorders (Carapetis et al., 2005; Bisno, 1991; Potter et al., 1978; Bessen et al., 1989; Nordstrand et al., 1999; Couser, 1999). Rheumatic fever, as well as the invasive infections, result in striking annual morbidity and lethality rates (estimated 1.78 million cases and 517000 deaths per year) (Carapetis et al., 2005; Cunningham, 2000; Wahl et al., 2007; Stevens, 1996).

Serological differentiation of the surface attached, antigenically variable, major virulence factor $\mathrm{M}$ protein allowed the discrimination of up to 80 different serotypes 
among the GAS (Lancefield, 1928, 1962). More recently, genotyping of the M-protein-encoding emm gene was established (Podbielski et al., 1991, 1994). This method currently permits the identification of more than $200 \mathrm{~S}$. pyogenes emm genotypes (emm types) as listed in the $S$. pyogenes emm sequence database of the Center for Disease Control and Prevention (http://www.cdc.gov/ncidod/ biotech/strep/strepblast.htm; Li et al., 2003). Recent studies performed in industrialized countries similarly demonstrated emm1, emm3, emm12 and emm28 as the most prevalent $\mathrm{emm}$ types associated with around $50 \%$ of all clinical cases (Wahl et al., 2007; Brandt et al., 2001; LucaHarari et al., 2008; Vlaminckx et al., 2004).

Genes encoding virulence factors and regulators, e.g. the major fibronectin-binding proteins $\mathrm{F} 1$ and 2 (prtF1, prtF2), the pilus-associated proteins $(c p a, f c t A, f c t B)$ and the RofA/Nra regulators ( $r o f A, n r a$ ), are located in the FCT region of the S. pyogenes genome (Bessen \& Kalia, 2002; Ramachandran et al., 2004; Nakata et al., 2005; Mora et al., 2005). The encoded factors were shown to play a role in matrix protein binding and global virulence regulation in selected emm-type strains (Manetti et al., 2007; Abbot et al., 2007; Podbielski et al., 1999; Kreikemeyer et al., 1995, 2005, 2007). This region is variable in both gene content and sequence. Currently nine different FCT types can be distinguished (Kratovac et al., 2007; Falugi et al., 2008). These FCT types have been demonstrated to include different subsets of emm genotypes. It is still not known whether this association consistently applies to multiple strains of given emm types or if the association could be extended to the anatomical sites from which the isolates originated.

Biofilms are known to be the most ubiquitous form of life among micro-organisms and are of special significance in medicine (Hall-Stoodley et al., 2004; Parsek \& Singh, 2003; Donlan \& Costerton, 2002; Cvitkovitch et al., 2003). Bacteria forming biofilm structures show increased resistance against environmental influences compared to planktonic cells of the same species (Donlan \& Costerton, 2002; Baldassarri et al., 2006; Conley et al., 2003; Gualtieri et al., 2006). S. pyogenes was shown to form mono-species biofilms in vivo and under in vitro conditions. The ability to build a biofilm apparently varies between individual strains and, so far, has not been associated with the above-mentioned typing schemes (Potter et al., 1978; Conley et al., 2003; Baldassarri et al., 2006; Akiyama et al., 2003; Lembke et al., 2006; Takemura et al., 2004). Courtney and co-workers recently showed that the complex formation of $\mathrm{M}$ protein with lipoteichoic acid is crucial for surface hydrophobicity and biofilm formation (Courtney et al., 2009). Furthermore the pilus-associated genes were demonstrated to be involved in biofilm formation in the M1 SF370 strain belonging to the FCT 2 type (Manetti et al., 2007). However, the association between pilus and biofilm formation has not been studied on a broader scale.

The present study investigated a collection of $183 \mathrm{~S}$. pyogenes isolates prospectively isolated from 2001 to 2006 in the University Hospital of Rostock (Germany). Their emm and FCT types, as well as their PFGE and antibioticresistance patterns, were assessed to classify them and to get information about their population diversity. We compared these data for all isolates to figure out relationships between the screened features. In addition, we measured biofilm formation with selected isolates from the identified clonal strains using different culture media. When comparing this biological function with the abovementioned features, new associations could be established. They appear to be representative for a multitude of strains of given emm or FCT types rather than for the clinical background of such strains. Moreover, on the basis of this study we stress the necessity for rigorous standards to determine (i) how isolates should be collected, (ii) how the clinical background is recorded and (iii) which parameters need to be determined to perform epidemiological studies with S. pyogenes.

\section{METHODS}

Isolates. The $S$. pyogenes isolates were collected prospectively from patients of the University Hospital, Rostock (Mecklenburg Vorpommern, Germany), from 2001 to 2006. The hospital functions as a centre for tertiary care with 1070 patient beds and serves approximately 500000 inhabitants. The patients represented three groups: (i) patients suffering from pharynx infections, including tonsillitis and tonsillar abscesses, (ii) patients suffering from skin infections at any location, including local abscesses, and (iii) patients with invasive diseases, i.e. the presence of bacteria at normally sterile anatomical sites. The $S$. pyogenes isolates were included in the collection according to the following criteria: (i) the originating persons had to be inpatients of the University Hospital, Rostock, (ii) the aetiopathology and class of infection had to be thoroughly stated in the accompanying documents, (iii) patients were not immunocompromised, (iv) isolates had to have been identified and processed in the diagnostic laboratory of the University Hospital, (v) S. pyogenes had to be the only pathogen in the specimen, (vi) only the first isolate per patient was included in the collection.

All isolates were differentiated as $\beta$-haemolytic streptococci of serological group A using the BBL Streptocard enzyme latex test agglutination kit and the API 20 Strep biochemical differentiation scheme (bioMérieux) according to the manufacturers' protocols. Isolates were aliquoted in Todd-Hewitt broth supplemented with $0.5 \%$ yeast extract [Todd-Hewitt yeast (THY) medium; Oxoid)] and $20 \%$ (v/v) glycerol, after a maximum two passages on Columbia sheep blood agar in the diagnostic laboratory of the University Hospital, Rostock, and were subsequently stored at $-80^{\circ} \mathrm{C}$. All subsequent analytical procedures where performed by starting a preculture in THY broth using a storage culture aliquot to exclude selection effects due to extensive passaging $\left(37{ }^{\circ} \mathrm{C}, 5 \% \mathrm{CO}_{2} / 20 \%\right.$ $\mathrm{O}_{2}$ ). For molecular analysis cells were cultured in THY broth at the above described conditions until an $\mathrm{OD}_{600}$ of 0.6 was reached. For biofilm analyses, cells were cultured in C medium (Manetti et al., 2007), tryptic soy broth (TSB; Oxoid), THY medium (Oxoid) and brain heart infusion (BHI) medium (Oxoid) for $24 \mathrm{~h}$ at $37{ }^{\circ} \mathrm{C}, 5 \%$ $\mathrm{CO}_{2}$. If not stated otherwise, all different batches of media and the diagnostic reagents were purchased from Becton Dickinson.

Antibiotic-resistance test. The antibiotic susceptibility of all isolates was assessed by agar diffusion tests according to Clinical and Laboratory Standards Institute (CLSI) directives M100-S16 and 
M2-A9 (CLSI, 2006a, b). Briefly, all isolates were suspended in $100 \mu \mathrm{l}$ $0.9 \% \mathrm{NaCl}$ to reach a final $\mathrm{OD}_{600}$ of 0.5 . Subsequently isolates were plated on Mueller-Hinton agar supplemented with $5 \%$ sheep blood. Antibiotic discs contained penicillin $(10 \mu \mathrm{g})$, vancomycin $(30 \mu \mathrm{g})$, erythromycin $(15 \mu \mathrm{g})$, clindamycin $(2 \mu \mathrm{g})$, levofloxacin $(5 \mu \mathrm{g})$, tetracycline $(30 \mu \mathrm{g})$ and gentamicin $(30 \mu \mathrm{g})$. Antibiotic discs were stamped on prepared plates using a $\mathrm{BD}$ disc dispenser. The plates were incubated for $18-24 \mathrm{~h}$ at $35{ }^{\circ} \mathrm{C}$ in $20 \% \quad \mathrm{O}_{2} / 5 \% \mathrm{CO}_{2}$ atmosphere. Inhibition zones were evaluated and interpreted according to CLSI standards.

PFGE. PFGE analysis was performed according to established protocols (Miranda et al., 1991) with minor modifications. Briefly, cells were cultured in $5 \mathrm{ml}$ THY medium overnight. Approximately $10^{7} \mathrm{~S}$. pyogenes cells were transferred into casting wells and covered with agarose (Plaque GP agarose; Biozym). The resulting blocks were then exposed to mutanolysin, lysozyme (both Difco) and phagelysin C (kindly provided by Vincent Fischetti, Rockefeller University, New York, USA, and Daniel Nelson, University of Maryland, Rockville, MA, USA) at $37{ }^{\circ} \mathrm{C}$ for $5 \mathrm{~h}$. The liberated genomic DNA was digested overnight using SmaI restriction enzyme. After digestion the blocks were cut into plugs of $3 \times 5 \times 1 \mathrm{~mm}$ and embedded into $1 \%$ running gels with $0.5 \%$ Tris/borate/EDTA buffer $(\mathrm{pH} 8)$. The PFGE was performed with a CHEF-DRIII electrophoresis system (Bio-Rad Laboratories) for $22 \mathrm{~h}$ using the following settings: initial switch time, $5 \mathrm{~s}$; final switch time, $35 \mathrm{~s}$, voltage, $5.5 \mathrm{~V} \mathrm{~cm}^{-1}$; included angle, $120^{\circ}$. Gels were stained with ethidium bromide, and images captured with the Gel Jet imager (Intas Science Imaging Instruments) and analysed with GelCompar II software using the UPGMA algorithm with Dice coefficients (Applied Maths) (Chiou et al., 2004).

DNA extraction and FCT typing. Purification of chromosomal DNA from GAS was performed according to the protocol of Cleary et al. (1988). The emm typing was performed according to established instructions using 'all emm' primer sequences (Beall et al., 1996; Podbielski et al., 1999). PCR products were purified with the PCR-clean-up system (Promega) and sequenced on both strands. Sequencing was repeated on two independent occasions using the ABI Prism 310 genetic analyzer (Applied Biosystems). The resulting sequences allowed read lengths of up to 1000 nucleotides, thus guaranteeing sufficient overlap for whole gene sequence assembly. The compiled sequences were compared to the CDC emm database (http://www.cdc.gov/ncidod/biotech/strep/strepindex.htm). Degrees of homology and emm types were determined as described by Beall et al. (1996).

FCT typing was performed for each strain on two independent occasions using chromosomal DNA purified as mentioned above. Sequences from genes located in the FCT region were amplified by 34 cycles of conventional PCR using 60 pmol primers (listed in Supplementary Fig. S1 available with the online journal).

Following gel electrophoresis separation and detection, for each PCR the presence or absence of the expected gene-specific products were recorded according to the protocols of Kratovac et al. (2007) and Falugi et al. (2008). The resulting deduced gene patterns were assigned to the established FCT types (Supplementary Fig. S1 available with the online journal).

Biofilm analysis. Representative isolates from each newly identified clonal strain were directly inoculated from the freezer stocks into $10 \mathrm{ml}$ THY medium pre-culture and incubated overnight at $37^{\circ} \mathrm{C}$. A $50 \mu \mathrm{l}$ aliquot of these pre-cultures was added to $950 \mu \mathrm{l}$ fresh medium (C medium, BHI, TSB, THY) present in the uncoated wells of 24-well polystyrene plates. Plates were incubated at $37{ }^{\circ} \mathrm{C}$ for $24 \mathrm{~h}$. The medium was removed, wells were washed twice with PBS and the remaining adherent bacteria were stained with crystal violet $[0.2 \%$ in double deionized $\mathrm{H}_{2} \mathrm{O}\left(\mathrm{ddH}_{2} \mathrm{O}\right)$, room temperature, 15 min]. After removing the staining solution, the wells were washed twice with PBS. Remaining crystal violet firmly associated to the cells was dissolved with $1 \mathrm{ml}$ aqueous $1 \%$ SDS solution shaking for $10 \mathrm{~min}$. Quantification was achieved by diluting the solution $1: 10$ in $\mathrm{ddH}_{2} \mathrm{O}$ and subsequently measuring the $\mathrm{OD}_{540}$ (Manetti et al., 2007). Analyses with TSB and THY medium were performed in triplicate on two occasions (biological replicates) with each representative isolate (data not shown). Experiments with $\mathrm{C}$ medium and BHI broth were repeated on four independent occasions. Results for the two latter media are shown as mean values for each representative isolate. Moreover, selected isolates of each FCT type were checked on five independent occasions (biological replicates) using at least three different medium batches (Supplementary Fig. S2 available with the online journal).

\section{RESULTS AND DISCUSSION}

\section{Clinical data}

During the study period 2001-2006, 183 out of a total number of 217 S. pyogenes isolates fulfilled the inclusion criteria of this study and were included in the strain collection. The annual distribution of the collected strains was as follows: 2001, 19; 2002, 32; 2003, 40; 2004, 30; 2005,$41 ; 2006,21$. The isolates originated from upper respiratory tract infections (91 strains, $50 \%$ ), skin infections (71 strains, 39\%) and invasive diseases (21 strains, 11\%) (Supplementary Table S1 available with the online journal). Of the patients $109(60 \%)$ were male, $74(40 \%)$ female. With respect to age distribution for all cases the peak incidence was for patients from 0 to 10 years. The incidence sharply decreased in the middle decades of age and only slightly increased again for patients above 70 years (Fig. 1). With respect to infection sites, the rate of upper respiratory tract infections decreased whereas skin and invasive infection rates increased for elderly patients (Fig. 1).

Several epidemiological conclusions can be drawn from the data above. First, the clinical background of our collection (Fig. 1), reflects the regional ratio between $S$. pyogenes upper respiratory tract, skin and invasive infections leading to admission in a tertiary care hospital. Second, within the local population S. pyogenes infections affected more men than women. This difference was predominantly due to a higher rate of skin infections in our male patients. This finding is consistent with most recent observations from several European countries (Lamagni et al., 2008).

Third, with respect to the age of our patients, the incidence of all $S$. pyogenes infections decreased over life time. This observation is different from other studies that predominantly included patients suffering from invasive diseases (Darenberg et al., 2007; Ekelund et al., 2005; Luca-Harari et al., 2008). The relatively small number of invasive disease cases among our patients also showed a positive association with increasing age. According to the present investigation, the number of pharyngitis patients sharply drops above the age of 40, while skin infections become predominant. Finally, there could be regional differences in the age distribution, since, similar to the present, data a prevalence 


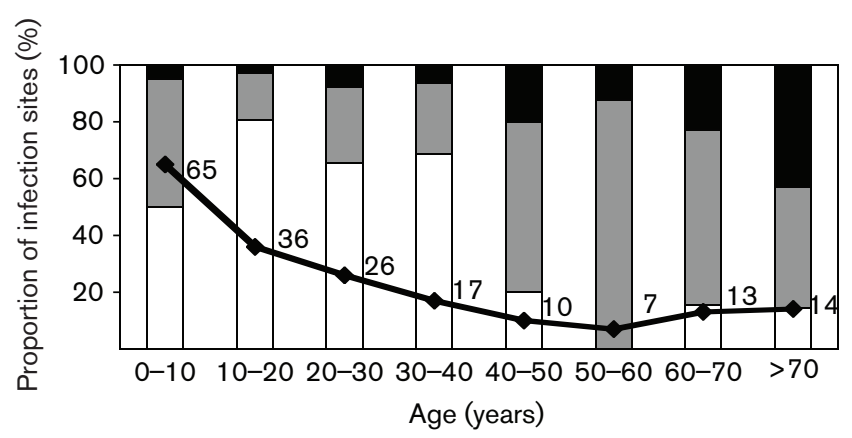

Fig. 1. Clinical background of the patients providing the isolates in the collection - age distribution of patients relative to infection types caused by the $S$. pyogenes isolates. The black line shows the distribution for all recorded cases complemented with overall numbers of isolates for each age group. Bars represent the percentage of infection sites relative to the overall number of strains isolated from individual age groups. White bars, throat infection; grey bars, skin infection; black bars, invasive infection.

peak in patients below 40 years was also observed in two other Dutch and German studies (Vlaminckx et al., 2004; Wahl et al., 2007).

\section{emm typing}

emm typing identified 25 different emm genotypes in 183 isolates (Table 1, Fig. 2). The most frequently identified emm types were emm28 (24\%), emm12 (11\%), emm1 (11\%), emm4 (8\%), emm2 (7\%) and emm89 (7\%). Altogether, these six emm types accounted for $68 \%$ of the isolates. The diversity and frequency of identified emm types among our isolates resembles those from comparable studies in Germany, Denmark, Sweden and Poland (Darenberg et al., 2007; Ekelund et al., 2005; Luca-Harari et al., 2008; Reinert et al., 2004; Szczypa et al., 2004; Wahl et al., 2007). Eight out of the eleven most frequently found emm types in those studies were also among the ten most frequent $\mathrm{emm}$ types in our study. This illustrates a wellknown regional geographical stability of certain emm types over a limited time period. Over the sampling period of 6 years, no change of the prevailing emm types was observed. Compared to other studies (Colman et al., 1993), this unexpected result could be due to the small sample size or the short sampling period. Different from former studies, we could not demonstrate an association between isolation sites and emm types among our strains (Fig. 2). This result is beyond doubt for clonal isolates from different infection sites. However, for the majority of individual strains the result could have been affected by the sampling size in the present study.

\section{Antibiotic resistance}

Analysis of the antibiotic-susceptibility profiles of all the isolates showed 41 of $183(22 \%)$ isolates to be resistant
Table 1. emm types identified in the present clinical $S$. pyogenes strain collection

\begin{tabular}{|l|c|}
\hline emm type & No. \\
\hline emm28 & 44 \\
emm12 & 21 \\
emm1 & 20 \\
emm 4 & 15 \\
emm2 & 13 \\
emm89 & 14 \\
emm6 & 8 \\
emm77 & 8 \\
emm75 & 8 \\
emm118 & 7 \\
emm78 & 7 \\
emm58 & 6 \\
emm3 & 6 \\
emm 44 & 4 \\
emm94 & 4 \\
st794 & 2 \\
emm109 & 2 \\
emm11 & 2 \\
emm15 & 2 \\
emm49 & 2 \\
emm67 & 2 \\
emm81 & 2 \\
emm83 & 2 \\
st3765 & 1 \\
st 661 & 1 \\
\hline
\end{tabular}

against one or more antibiotics (Table 2). The most frequent unique resistance traits were those directed against erythromycin (ten isolates), tetracycline (eight isolates), levofloxacin (six isolates) and gentamicin (six isolates). Less frequently we identified combined antibiotic resistance traits against erythromycin/tetracycline (five isolates), erythromycin/levofloxacin (three isolates), levofloxacin/tetracycline (two isolates) and gentamicin/levofloxacin (one isolate) (Table 2). The recorded erythromycin resistance rate of $9.8 \%$ was below the $14 \%$ rate recently reported for juvenile and adult Germans (Reinert et al., 2003, 2004; Sauermann et al., 2003). This could be due to a sampling area mostly in the western and southern parts of the country in the three studies. Correspondingly, erythromycin resistance rates among patients from geographically less distant countries such as Denmark and Poland ranged between 3 and 12\% (Luca-Harari et al., 2008; Szczypa et al., 2004, 2006).

The small but noticeable levofloxacin-resistance rate among our isolates is remarkable. This resistance trait was extremely rare in European isolates during the sampling period (0-0.2\%) (Biedenbach et al., 2006; Cantón et al., 2002; Sauermann et al., 2003). Locally higher rates were associated with the occurrence of specific GAS emm6 clones (Albertí et al., 2005; Orscheln et al., 2005). Among our 11 levofloxacin-resistant strains, only 2 carried the emm 6 gene and all isolates belonged to different 


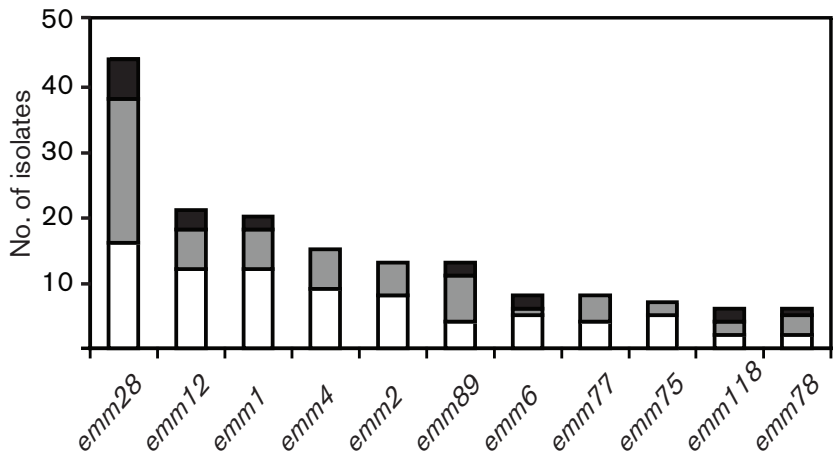

Fig. 2. emm types of isolated $S$. pyogenes strains relative to infection sites. The overall number of isolates from each emm type is shown. White bars, the number of isolates associated with throat infections; grey bars, the number of isolates associated with skin infections; black bars, the number of isolates associated with invasive infections.

clonal categories, indicating different and independent mutation or selection events as a basis for the observed prevalence of levofloxacin resistance and strain diversity.

As expected, all isolates were found to be susceptible to penicillin and vancomycin under the tested conditions. Depending on the infection site, we detected different proportions of resistant isolates (Fig. 3a). While $33 \%$ of all isolates obtained from patients with respiratory diseases showed at least one resistance trait, only $19 \%$ of the invasive isolates and $10 \%$ of all skin isolates did so. Of note, our pharyngitis isolates displayed a threefold higher erythromycin-resistance rate than the simultaneously collected skin isolates, while the clonal relatedness between the resistant isolates was equally low in both groups (Supplementary Table S1 available with the online journal). When comparing isolates from invasive diseases and asymptomatic pharyngeal carriers, Baldassarri and co-workers made a similar observation (Baldassarri et al., 2007). In both studies, a co-segregation of macrolideresistance genes and site-specific, but varying, virulence traits as described by Creti et al. (2005), Haller et al. (2005), Robinson et al. (2006) and Spinaci et al. (2004) could be a possible explanation.

The proportions of isolates resistant to one or more antibiotics were found to vary among different $\mathrm{emm}$ types (Table 2). For instance only $14 \%$ of all emm 28 and emm 12 isolates displayed any antibiotic resistance. The percentages for the emm1, emm4 and emm77 were 35, 47 and 50\%, respectively (Table 2 ).

\section{PFGE typing}

PFGE typing performed for all isolates resulted in the identification of 94 PFGE band patterns (Supplementary Table S1 available with the online journal). Seven isolates were not assignable because no band pattern could be achieved using the protocol described in Methods. According to the commercial analysis program, 82 isolates showed a similar or identical PFGE band pattern to other isolates.

The diversity of PFGE patterns varies between the different emm types and within the same emm type. Among the 44 emm28 isolates we observed 21 different PFGE patterns. Thus $50 \%$ of the isolates shared their PFGE pattern with another isolate (i.e. $50 \%$ of emm 28 isolates are clonally related) (Supplementary Table S1 available with the online

\section{Table 2. Antibiotic-resistance analysis}

The number of strains with specific antibiotic-resistance traits is shown in relation to their emm type. All, specific antibiotic-resistance traits among all tested S. pyogenes isolates; emm type, absolute numbers and ratios of resistant isolates relative to their emm types; resistant, percentage of antibiotic-resistant isolates among all tested streptococci or strains of a given emm type.

\begin{tabular}{|c|c|c|c|c|c|c|c|c|c|c|c|c|c|c|}
\hline \multirow[t]{2}{*}{ Antibiotic resistance } & \multicolumn{14}{|c|}{ emm type } \\
\hline & All & emm28 & emm12 & emm1 & emm4 & emm89 & emm2 & emm6 & emm77 & emm78 & emm58 & emm94 & st794 & emm81 \\
\hline Erythromycin & 10 & 1 & & 2 & 4 & 1 & & 1 & & & & & & 1 \\
\hline Tetracycline & 8 & 1 & & 2 & 2 & & 1 & & 1 & 1 & & & & \\
\hline Levofloxacin & 6 & 1 & 1 & 2 & & & & 2 & & & & & & \\
\hline Gentamicin & 6 & & 1 & 1 & & 1 & 1 & & 1 & & & 1 & & \\
\hline Penicillin G & 0 & & & & & & & & & & & & & \\
\hline Clindamycin & 0 & & & & & & & & & & & & & \\
\hline Vancomycin & 0 & & & & & & & & & & & & & \\
\hline Erythromycin/tetracycline & 5 & & & & & & & & 1 & & 3 & & 1 & \\
\hline Erythromycin/levofloxacin & 3 & 1 & 1 & & 1 & & & & & & & & & \\
\hline Levofloxacin/tetracycline & 2 & 1 & & & & & & & 1 & & & & & \\
\hline Gentamicin/levofloxacin & 1 & 1 & & & & & & & & & & & & \\
\hline Total & 41 & 6 & 3 & 7 & 7 & 2 & 2 & 3 & 4 & 1 & 3 & 1 & 1 & 1 \\
\hline Resistant (\%) & $22 \%$ & $14 \%$ & $14 \%$ & $35 \%$ & $47 \%$ & $14 \%$ & $15 \%$ & $38 \%$ & $50 \%$ & $17 \%$ & $75 \%$ & $50 \%$ & $50 \%$ & $100 \%$ \\
\hline
\end{tabular}


(a)

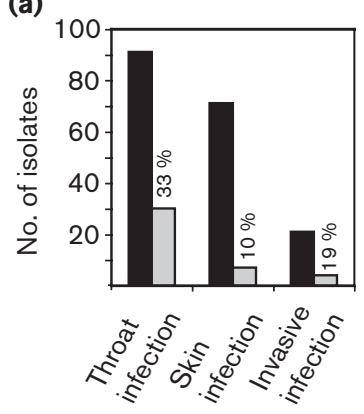

(b)

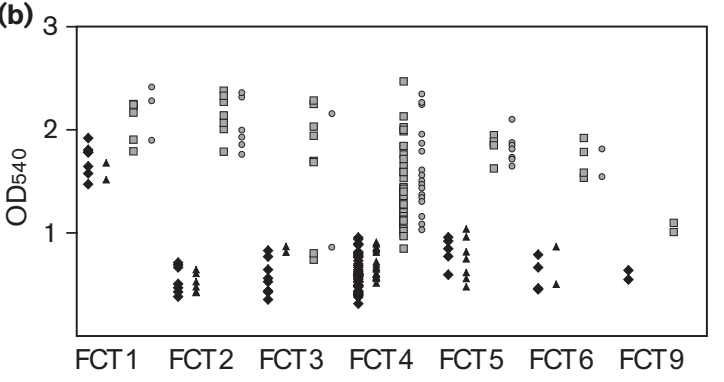

Fig. 3. Antibiotic resistance analysis. (a) Numbers of resistant isolates relative to infection sites. Black bars, all isolates from one infection site; grey bars, antibiotic-resistant isolates from one infection site. (b) Biofilm formation capacity of antibiotic-sensitive and antibiotic-resistant isolates. C, C medium cultures, antibiotic-sensitive isolates; $\boldsymbol{\Delta}, \mathrm{C}$ medium cultures, antibiotic-resistant isolates; $\square, \mathrm{BHI}$ cultures, antibiotic-sensitive isolates; , $\mathrm{BHI}$ cultures, antibiotic-resistant isolates. Isolates are sorted according to their association with FCT types 1-9. journal). For the $21 \mathrm{emm} 12,20 \mathrm{emm} 1$ and $13 \mathrm{emm} 2$ isolates we found a lower diversity of 5 PFGE patterns (76\% clonally related strains), 6 PFGE patterns ( $70 \%$ clonally related strains) and 4 PFGE patterns (69\% clonally related strains), respectively. A higher diversity was detected for 15 emm 4 and $14 \mathrm{emm} 89$ isolates with 10 PFGE patterns (13\% clonally related strains) and 7 PFGE patterns (46\% clonally related strains), respectively.

Isolates with frequently detected emm types typically displayed one major PFGE pattern (majority of isolates). The other PFGE patterns were only found for one or a few isolates. For instance, 14 of $42 \mathrm{emm} 28$ isolates displayed the PFGE pattern 7, while only 4 isolates were found to share the PFGE pattern 4. Of note, isolates associated with the same clonal group were found to originate from different infection sites. This indicates that even genetically identical $S$. pyogenes showed poor tissue preferences.

\section{FCT typing}

Most recently, the major fibronectin- and collagen-binding proteins encoded by the FCT region were suggested to be involved in S. pyogenes tissue specificity (Abbot et al., 2007; Lizano et al., 2007; Manetti et al., 2007). Arguments are based on their specific functions, variable presence and sequence in different GAS strains, as well as a temperaturedependent expression profile consistent with skin infection conditions (Nakata et al., 2009).

Several attempts to demonstrate such correlations have been made by screening clinical $S$. pyogenes isolates for the existence of selected FCT region genes (Kreikemeyer et al., 2004; Baldassarri et al., 2007; Musumeci et al., 2003). Overall these attempts did not result in clear conclusions concerning the role of individual FCT region factors for infection targeting. Consequently, instead of identifying selected FCT genes the FCT types were determined for 183 isolates. The most frequent FCT type was FCT 4 detected in 105 isolates. The other FCT types were found as follows: FCT 1, 11 isolates; FCT 2, 22 isolates; FCT 3, 10 isolates; FCT 5, 15 isolates; FCT 6, 14 isolates; FCT 7, 0 isolates; FCT 8, 0 isolates; FCT 9, 6 isolates (Fig. 4). No FCT type showed an above-average association rate for a specific infection site (Fig. 4). Obviously no simple correlation exists between the presence of certain FCT region types and the anatomical site from where GAS isolates originate. Since the expression of the FCT region factors is controlled by several regulators in an FCT typeor even strain-dependent fashion, and involves other peculiar regulatory mechanisms (Nakata et al., 2005, 2009; Kreikemeyer et al., 2007), correlating the expression levels with anatomical sites could perhaps give a better clue.

FCT types 3 and 4 included the highest quantity of different $\mathrm{emm}$ types ( 8 and $12 \mathrm{emm}$ types, respectively, Fig. $4 \mathrm{~b})$. Furthermore, we identified some emm type strains that had not been FCT-typed by Kratovac et al. (2007) (emm78, FCT 4; st794, FCT 3 and FCT 4; stG61, FCT 6). With respect to clonal groups as defined by identical PFGE patterns and emm types, again the majority of such clonal groups clustered in FCT type 4. Of note, we found five isolates that were associated with other FCT types than the majority of isolates with the same emm type (emm28 $2 \times$ FCT 2, emm 75 - FCT 4, emm12 - FCT 3, emm 118 FCT 1). Also the isolates of the newly defined emm type st794 belonged to FCT types 3 and 4 (Fig. 4b).

Another still unanswered question is what is the potential contribution of the FCT region factors to $S$. pyogenes persistence after an acute infection? This question cannot be directly addressed using our data, since our collection did not include strains from asymptomatic carriers. However, GAS growth in superficial and intra-tissue mono-species biofilms has been used as an alternative explanation for antibiotic therapy failure and persistence (Baldassarri et al., 2006; Conley et al., 2003).

\section{Classification of strains}

Next, an assignment of isolates was carried out by integrating all previously reported results. The number of isolates - according to different collection criteria - showing distinct properties for at least one of the three typing parameters (emm type, PFGE type and FCT type) was 95 . This corresponds to $52 \%$ of the complete strain collection. If antibiotic resistance was added as a 
(a)

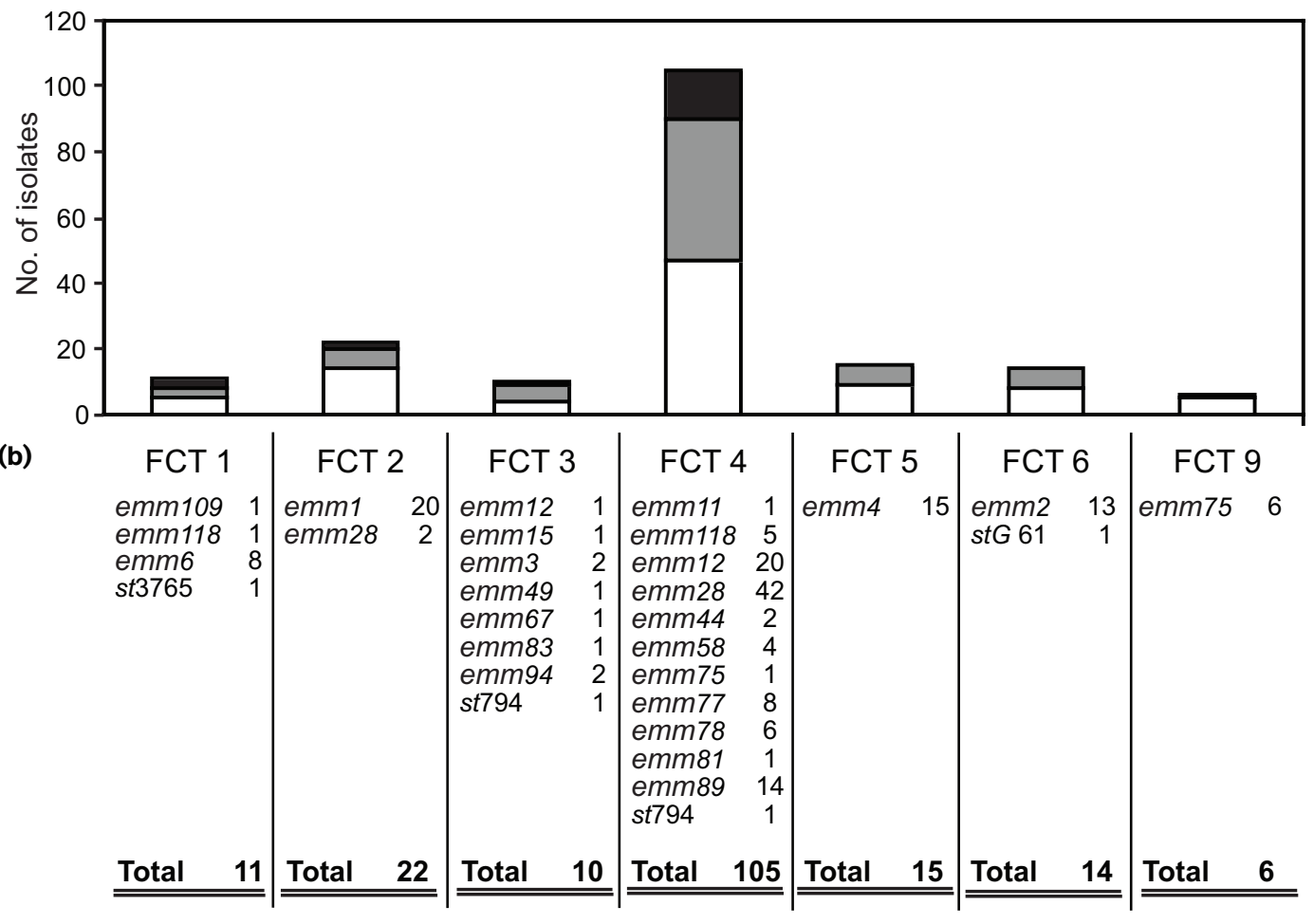

Fig. 4. FCT types of isolated S. pyogenes isolates relative to infection sites and emm types. (a) FCT types relative to infection sites. The total number of isolates belonging to individual FCT types is shown as composite bars. White bars, throat infections; grey bars, skin infections; black bars, invasive infections. (b) FCT types relative to emm types. Below the individual FCT types the number of isolates belonging to specific emm types as well as the overall number of isolates of this FCT type is shown.

distinction criterion, the total number of unique isolates increased to 113 , i.e. $62 \%$ of the total number of isolates. A total of 66 isolates were recognized to have characteristics identical to one of the 113 distinct strains and thus were regarded as clonally related to one of these distinct isolates (Supplementary Table S1 available with the online journal). Four isolates could not be assigned to either group because of incomplete typing results. According to the now defined classification directives we identified 40 of 41 antibiotic resistant isolates as distinctive. This shows a much stronger strain diversity of resistant isolates (only $2.5 \%$ clonally related strains) compared to susceptible isolates [73 distinct isolates, 65 clonally related isolates ( $47 \%$ clonally related strains)].

\section{Biofilm formation}

A role of the FCT 2-encoded pilus proteins in biofilm growth was recently demonstrated (Manetti et al., 2007). For this reason, and in order to establish biofilm testing as a novel epidemiological marker for $S$. pyogenes, we tested the biofilm formation capacity of representative isolates. We found sufficient data to support a type-specific function of FCT-region factors in biofilm development and/or maturation. However, this correlation was superimposed in the majority of cases by culture medium effects (Fig. 5). In each of the earlier publications on S. pyogenes biofilm formation, different culture media were used. In this study we tested C medium, THY, TSB and BHI media to compare their influence on biofilm formation measured using a plate assay after $24 \mathrm{~h}$ incubation periods. In the following section only the results for $\mathrm{C}$ medium and $\mathrm{BHI}$ broth are shown (Fig. 5a). Biofilm growth was tested for each of the 183 isolates. Within the typical range of strains with the same FCT type, the clonal isolates formed biofilms like the corresponding distinct strains. In order to exclude statistical bias due to clonal isolates, only the results obtained with the 113 distinct isolates are shown in this paragraph.

Biofilm formation experiments using $\mathrm{C}$ medium generally showed smaller biofilm masses for the majority of isolates than experiments using BHI medium, except for FCT 1 strains (see below). Results for strains cultured in THY medium or TSB were generally ranged in-between the results from the other two media.

As already seen for the FCT types, biofilm formation behaviour was found to be independent from the 


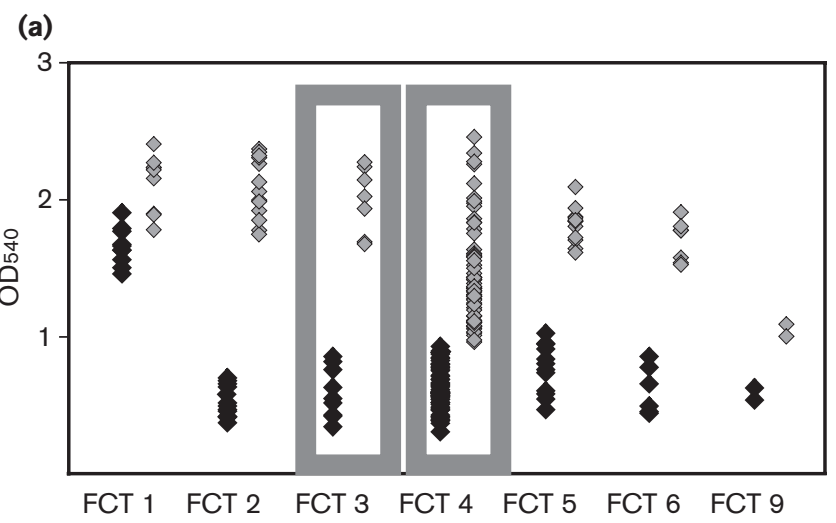

(b) (i)

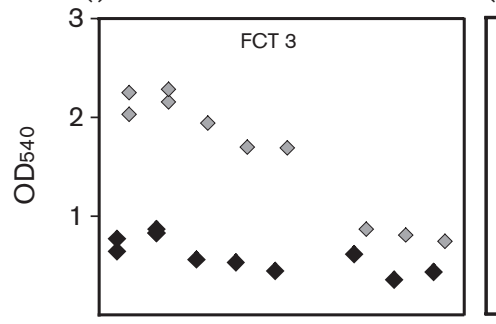

(ii)

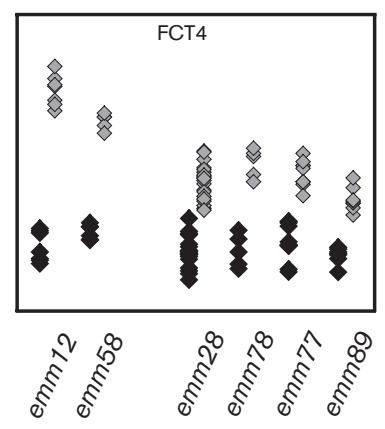

(c)
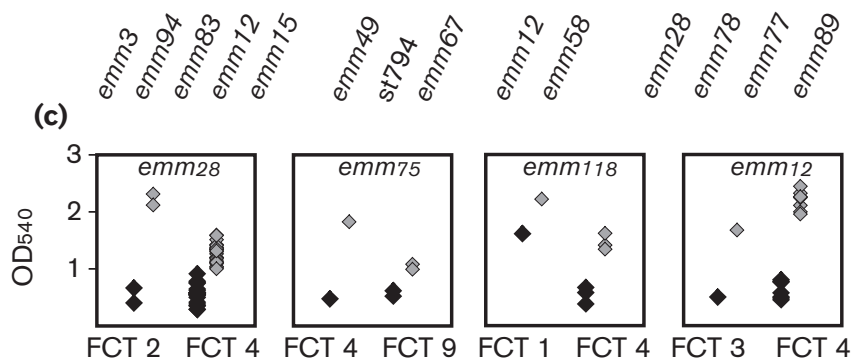

Fig. 5. Biofilm formation of unique $S$. pyogenes strains relative to culture media, FCT types and emm types. (a) Biofilm formation relative to culture medium and FCT types. On four independent occasions the biofilm-forming capacities of representative isolates from the 113 unique $S$. pyogenes strains defined in this study were measured after $24 \mathrm{~h}$ culture in $\mathrm{C}$ medium and $\mathrm{BHI}$ broth. Biofilm mass was assessed by crystal violet staining and subsequent determination of the $\mathrm{OD}_{540}$. The resulting mean values are shown after ordering them relative to the FTC types of the tested isolates. (b) Biofilm formation of representative FCT types 3 and 4 isolates from the unique $S$. pyogenes strains relative to culture media and emm types. Individual $S$. pyogenes strains were selected and processed as described above. As further detail for Fig. 5(a), the representative isolates were additionally ordered with respect to their emm types. Among both FCT types there are two groups of emm type strains, which displayed either marked or little difference in biofilm formation when cultured in the two media. (c) Biofilm formation of $S$. pyogenes isolates belonging to identical emm types but different FCT types. The $S$. pyogenes isolates were processed as described for Fig. 5(a). Comparable to Fig. 5(b), different strains belonging to one emm type displayed marked or little difference in biofilm formation when cultured in the two media. Of note, the individual isolates built biofilm masses like other isolates with different emm types but identical FCT types. $\diamond$, medium cultures; $>$ BHI broth cultures. anatomical site of isolation. Also the antibiotic-resistance patterns could not be associated with a special capacity to form 'in vitro' biofilms (Fig. 3b). We found selected isolates to tolerate higher antibiotic concentrations of penicillin, erythromycin and tetracycline added to mature 'in vitro' biofilms independent from their individual antibioticresistance profile (unpublished data). These results indicate the lack of genetic linkage between genes responsible for biofilm formation and conventionally determined antibiotic resistance, and simultaneously, the protection against defined antibiotic activities among biofilm-organized cells. Our data differ from those of Baldassarri et al. (2006) who demonstrated the ability to grow large biofilm masses was associated with a lack of erythromycin resistance in planktonic bacteria. When looking at the results of these authors, strains without protein F1 genes were more strongly associated with biofilm-formation capacity, indicating that Baldassarri and colleagues could have detected a special subset of $S$. pyogenes strains (Baldassarri et al., 2006).

However, we identified an association between several FCT types and corresponding biofilm-formation behaviour. FCT 1-associated strains were the only ones that showed biofilm formation irrespective of the culture media used (Fig. 5a). This observation should be kept in mind when interpreting the, so far, single transcriptome data on $S$. pyogenes biofilms obtained with an emm6 FCT type 1 strain (Cho \& Caparon, 2005). In that study, Caparon and coworkers found the emm gene but no FCT region genes to be markers for biofilm formation. For a more representative view on the biofilm-relevant GAS transcriptomes, isolates from the much more frequent FCT types 3 and 4 should be analysed. Yet, biofilm formation and maturation is a multi-step process that obviously follows different rules in various $S$. pyogenes strains. Thus, even if FCT region factors obviously contribute to this feature, a complementary function is exerted by other factors such as M proteins in subsets of $S$. pyogenes strains. Experimental support for such a complementary function was most recently provided by work from Courtney et al. (2009). They proved that complexes of $\mathrm{M}$ protein with lipoteichoic acid contribute to biofilm formation (Courtney et al., 2009).

Strains belonging to FCT types 2, 5 and 6 (Fig. 5a) displayed biofilm development depending on the type of culture medium used for the assay. Representatives of FCT type 9 formed weak biofilm in all tested media (Fig. 5a).

While all strains with FCT types 1, 2, 5, 6 and 9 showed similar biofilm-formation rates for a given FCT type, strains with FCT types 3 and 4 displayed a heterogeneous biofilm growth within their FCT groups (Fig. 5a). One group of FCT 3 type strains (emm12, emm15, emm3, emm83, emm94) formed biofilm when cultured in BHI medium but not in C-medium, while the other group (emm49, emm67, st794) were poor biofilm formers in both media (Fig. 5bi). For FCT 4 type strains the biofilm formation capacities were even more heterogeneous, 
displaying different combinations of high and low biofilm masses when grown in different media (Fig. 5bii, right).

Based on the limited number of isolates studied here the association between FCT types and biofilm-formation capacity was stronger than any association between $\mathrm{emm}$ type and biofilm. For example, emm 28 strains belonging to FCT 2 type behaved like other FCT 2 type strains, while all emm 28 strains belonging to FCT 4 type collectively showed a different biofilm formation behaviour (Fig. 5c). A similar observation was made for the emm75 strains and their association to FCT types 4 and 9, the emm12 strains and their FCT types 3 and 4, and the emm118 strains associated to FCT types 1 and 4 (Fig. 5c).

Overall, the majority of the tested strains formed biofilms depending on culture conditions. Only 11 FCT 1 type strains were found to consistently build biofilms independent of the employed media. Moreover only a minority of three FCT type 3, two FCT type 9 and eight FCT type 4 strains showed no biofilm formation under any culture condition.

On the basis of our data we suggest that $\mathrm{C}$ medium and BHI medium are the best media for comparative GAS biofilm investigations. This information should be very useful for other groups who would like to include biofilm formation as one parameter for future epidemiological studies. After elucidation of FCT types it is now clear which medium should be used. Our study has unequivocally demonstrated that the biofilm feature of GAS is stable, reliable and independent of biological replicate experiments and different medium batches (Supplementary Fig. S2 available with the online journal).

The major goal of the present investigation was to assess the association between established genetic traits $(\mathrm{emm}$ genotyping, PFGE and antibiotic resistance), novel genetic traits (FCT typing), functional features (biofilm formation capacities) and clinical background in a thoroughly defined collection of $S$. pyogenes hospital isolates. In conclusion, the rigorous inclusion criteria, as well as the thorough genetic and functional characterization of the strain collection, guaranteed that only isolates with an infectious background were taken into consideration. The thorough typing was particularly necessary since a strain collection from one hospital has a higher chance to predominantly include clonal isolates. An even number of samples per year as well as the identification of 113 distinct isolates (according to the typing criteria) among the different 183 isolates (whole strain collection) demonstrates the low degree of clonality in our clinical S. pyogenes collection. This further points towards the extent of diverse infectious contacts even in a comparatively small population of potential hosts. Determination of the clonality status of each isolate and the normalization of the analyses by operating with only one representative isolate per identified strain is a clear advantage over retrospective studies. Those previous studies often relied on strain collections sampled outside the analysing laboratory, frequently under varying criteria over the sampling period, not devoid of copy strains and with incomplete clinical background information. Furthermore, in our collection the passage numbers after sampling were recorded, which makes the interpretation of functional data, such as antibiotic resistance or biofilm formation, more reliable. In summary, our data indicate that FCT region typing extends the panel of methods for characterization of $S$. pyogenes strains. Moreover, biofilm formation capacity in defined media could be exploited as a new epidemiological marker.

\section{ACKNOWLEDGEMENTS}

The authors would like to thank Vince Fischetti (Rockefeller University, New York, USA) and Daniel Nelson (University of Maryland, Rockville, MA, USA) for kindly providing phagelysin C (PlyC). The work of A.P. and B.K. was supported by Bundesministerium für Bildung und Forschung (BMBF) grants BE031-03U213B, 0313936A and 0313979B as parts of the German PathoGenomik Competence Network, the BMBF SysMo 'Systems Biology of Microorganisms' programme and the ERA-NET 'PathoGenoMics' competence network, respectively. T. K. and B. K. further acknowledge support by the 2008 FORUN program of the Medical Faculty of the University of Rostock, Germany. All authors hereby state that no conflict of interest exists. G. G. and I. M. are holders of Novartis stock options.

\section{REFERENCES}

Abbot, E. L., Smith, W. D., Siou, G. P., Chiriboga, C., Smith, R. J., Wilson, J. A., Hirst, B. H. \& Kehoe, M. A. (2007). Pili mediate specific adhesion of Streptococcus pyogenes to human tonsil and skin. Cell Microbiol 9, 1822-1833.

Akiyama, H., Morizane, S., Yamasaki, O., Oono, T. \& Iwatsuki, K. (2003). Assessment of Streptococcus pyogenes microcolony formation in infected skin by confocal laser scanning microscopy. J Dermatol Sci 32, 193-199.

Alberti, S., Cortés, G., Garcia-Rey, C., Rubio, C., Baquero, F., GarcíaRodríguez, J. A., Bouza, E. \& Aguilar, L. (2005). Streptococcus pyogenes pharyngeal isolates with reduced susceptibility to ciprofloxacin in Spain: mechanisms of resistance and clonal diversity. Antimicrob Agents Chemother 49, 418-420.

Baldassarri, L., Creti, R., Recchia, S., Imperi, M., Facinelli, B., Giovanetti, E., Pataracchia, M., Alfarone, G. \& Orefici, G. (2006). Therapeutic failures of antibiotics used to treat macrolide-susceptible Streptococcus pyogenes infections may be due to biofilm formation. J Clin Microbiol 44, 2721-2727.

Baldassarri, L., Creti, R., Imperi, M., Recchia, S., Pataracchia, M. \& Orefici, G. (2007). Detection of genes encoding internalizationassociated proteins in Streptococcus pyogenes isolates from patients with invasive diseases and asymptomatic carriers. J Clin Microbiol 45, 1284-1287.

Beall, B., Facklam, R. \& Thompson, T. (1996). Sequencing emmspecific PCR products for routine and accurate typing of group A streptococci. J Clin Microbiol 34, 953-958.

Bessen, D. E. \& Kalia, A. (2002). Genomic localization of a T serotype locus to a recombinatorial zone encoding extracellular matrix-binding proteins in Streptococcus pyogenes. Infect Immun 70, 1159-1167.

Bessen, D., Jones, K. F. \& Fischetti, V. A. (1989). Evidence for two distinct classes of streptococcal $M$ protein and their relationship to rheumatic fever. J Exp Med 169, 269-283. 
Biedenbach, D. J., Toleman, M. A., Walsh, T. R. \& Jones, R. N. (2006). Characterization of fluoroquinolone-resistant $\beta$-hemolytic Streptococcus spp. isolated in North America and Europe including the first report of fluoroquinolone-resistant Streptococcus dysgalactiae subspecies equisimilis, report from the SENTRY Antimicrobial Surveillance Program (1997-2004). Diagn Microbiol Infect Dis 55, 119-127.

Bisno, A. L. (1991). Group A streptococcal infections and acute rheumatic fever. $N$ Engl J Med 325, 783-793.

Bisno, A. L. \& Stevens, D. L. (1996). Streptococcal infections of skin and soft tissues. $N$ Engl J Med 334, 240-245.

Brandt, C. M., Spellerberg, B., Honscha, M., Truong, N. D., Hoevener, B. \& Lütticken, R. (2001). Typing of Streptococcus pyogenes strains isolated from throat infections in the region of Aachen, Germany. Infection 29, 163-165.

Cantón, R., Loza, E., Morosini, M. I. \& Baquero, F. (2002). Antimicrobial resistance amongst isolates of Streptococcus pyogenes and Staphylococcus aureus in the PROTEKT antimicrobial surveillance programme during 1999-2000. J Antimicrob Chemother 50, 9-24.

Carapetis, J. R., Steer, A. C., Mulholland, E. K. \& Weber, M. (2005). The global burden of group A streptococcal diseases. Lancet Infect Dis 5, 685-694.

Chiou, C. S., Liao, T. L., Wang, T. H., Chang, H. L., Liao, J. C. \& Li, C. C. (2004). Epidemiology and molecular characterization of Streptococcus pyogenes recovered from scarlet fever patients in central Taiwan from 1996 to 1999. J Clin Microbiol 42, 3998-4006.

Cho, K. H. \& Caparon, M. G. (2005). Patterns of virulence gene expression differ between biofilm and tissue communities of Streptococcus pyogenes. Mol Microbiol 57, 1545-1556.

Cleary, P. P., Kaplan, E. L., Livdahl, C. \& Skjold, S. (1988). DNA fingerprints of Streptococcus pyogenes are M type specific. J Infect Dis 158, 1317-1323.

CLSI (2006a). Performance Standards for Antimicrobial Disk Susceptibility Tests, 9th edn, approved standard M2-A9. Wayne, PA: Clinical and Laboratory Standards Institute.

CLSI (2006b). Performance Standards for Antimicrobial Susceptibility Testing, 16th informational supplement, M100-S16. Wayne, PA: Clinical and Laboratory Standards Institute.

Colman, G., Tanna, A., Efstratiou, A. \& Gaworzewska, E. T. (1993). The serotypes of Streptococcus pyogenes present in Great Britain during 1980-1990 and their association with disease. J Med Microbiol 39, 165-178.

Conley, J., Olson, M. E., Cook, L. S., Ceri, H., Phan, V. \& Davies, H. D. (2003). Biofilm formation by group A streptococci: is there a relationship with treatment failure? J Clin Microbiol 41, 4043-4048.

Courtney, H. S., Ofek, I., Penfound, T., Nizet, V., Pence, M. A., Kreikemeyer, B., Podbielski, A., Hasty, D. L. \& Dale, J. B. (2009). Relationship between expression of the family of $\mathrm{M}$ proteins and lipoteichoic acid to hydrophobicity and biofilm formation in Streptococcus pyogenes. PLoS One 4, e4166.

Couser, W. G. (1999). Glomerulonephritis. Lancet 353, 1509-1515.

Creti, R., Gherardi, G., Imperi, M., von Hunolstein, C., Baldassarri, L., Pataracchia, M., Alfarone, G., Cardona, F., Dicuonzo, G. \& Orefici, G. (2005). Association of group A streptococcal emm types with virulence traits and macrolide-resistance genes is independent of the source of isolation. J Med Microbiol 54, 913-917.

Cunningham, M. W. (2000). Pathogenesis of group A streptococcal infections. Clin Microbiol Rev 13, 470-511.

Cvitkovitch, D. G., Li, Y. H. \& Ellen, R. P. (2003). Quorum sensing and biofilm formation in streptococcal infections. J Clin Invest 112, 16261632 .
Darenberg, J., Luca-Harari, B., Jasir, A., Sandgren, A., Pettersson, H., Schalén, C., Norgren, M., Romanus, V., Norrby-Teglund, A. \& Normark, B. H. (2007). Molecular and clinical characteristics of invasive group A streptococcal infection in Sweden. Clin Infect Dis 45, 450-458.

Donlan, R. M. \& Costerton, J. W. (2002). Biofilms: survival mechanisms of clinically relevant microorganisms. Clin Microbiol Rev 15, 167-193.

Ekelund, K., Darenberg, J., Norrby-Teglund, A., Hoffmann, S., Bang, D., Skinhøj, P. \& Konradsen, H. B. (2005). Variations in emm type among group A streptococcal isolates causing invasive or noninvasive infections in a nationwide study. J Clin Microbiol 43, 3101-3109.

Falugi, F., Zingaretti, C., Pinto, V., Mariani, M., Amodeo, L., Manetti, A. G. M., Capo, S., Musser, J. M., Orefici, G. \& other authors (2008). Sequence variation in group A Streptococcus pili and association of pilus backbone types with Lancefield T-serotypes. J Infect Dis 198, 1834-1841.

Gualtieri, M., Bastide, L., Villain-Guillot, P., Michaux-Charachon, S., Latouche, J. \& Leonetti, J. P. (2006). In vitro activity of a new antibacterial rhodamine derivative against Staphylococcus epidermidis biofilms. J Antimicrob Chemother 58, 778-783.

Hall-Stoodley, L., Costerton, J. W. \& Stoodley, P. (2004). Bacterial biofilms: from the natural environment to infectious diseases. Nat Rev Microbiol 2, 95-108.

Haller, M., Fluegge, K., Arri, S. J., Adams, B. \& Berner, R. (2005). Association between resistance to erythromycin and the presence of the fibronectin binding protein F1 gene, prtF1, in Streptococcus pyogenes isolates from German pediatric patients. Antimicrob Agents Chemother 49, 2990-2993.

Kratovac, Z., Manoharan, A., Luo, F., Lizano, S. \& Bessen, D. E. (2007). Population genetics and linkage analysis of loci within the FCT region of Streptococcus pyogenes. J Bacteriol 189, 1299-1310.

Kreikemeyer, B., Talay, S. R. \& Chhatwal, G. S. (1995). Characterization of a novel fibronectin- binding surface protein in group A streptococci. Mol Microbiol 17, 137-145.

Kreikemeyer, B., Klenk, M. \& Podbielski, A. (2004). The intracellular status of Streptococcus pyogenes: role of extracellular matrix-binding proteins and their regulation. Int J Med Microbiol 294, 177-188.

Kreikemeyer, B., Nakata, M., Oehmcke, S., Gschwendtner, C., Normann, J. \& Podbielski, A. (2005). Streptococcus pyogenes collagen type I-binding Cpa surface protein. Expression profile, binding characteristics, biological functions, and potential clinical impact. J Biol Chem 280, 33228-33239.

Kreikemeyer, B., Nakata, M., Köller, T., Hildisch, H., Kourakos, V., Standar, K., Kawabata, S., Glocker, M. O. \& Podbielski, A. (2007). The Streptococcus pyogenes serotype M49 Nra-Ralp3 transcriptional regulatory network and its control of virulence factor expression from the novel eno ralp3 epf sagA pathogenicity region. Infect Immun 75, 5698-5710.

Lamagni, T. L., Darenberg, J., Luca-Harari, B., Siljander, T., Efstratiou, A., Henriques-Normark, B., Vuopio-Varkila, J., Bouvet, A., Creti, R. \& other authors (2008). Epidemiology of severe Streptococcus pyogenes disease in Europe. J Clin Microbiol 46, 23592367.

Lancefield, R. C. (1928). The antigenic complex of Streptococcus haemolyticus. I. Demonstration of a type-specific substance in extracts of Streptococcus haemolyticus. J Exp Med 47, 91-103.

Lancefield, R. C. (1962). Current knowledge of type-specific M antigens of group A streptococci. J Immunol 89, 307-331.

Lembke, C., Podbielski, A., Hidalgo-Grass, C., Jonas, L., Hanski, E. \& Kreikemeyer, B. (2006). Characterization of biofilm formation by 
clinically relevant serotypes of group A streptococci. Appl Environ Microbiol 72, 2864-2875.

Li, Z., Sakota, V., Jackson, D., Franklin, A. R. \& Beall, B. (2003). Array of $\mathrm{M}$ protein gene subtypes in 1064 recent invasive group A Streptococcus isolates recovered from the active bacterial core surveillance. J Infect Dis 188, 1587-1592.

Lizano, S., Luo, F. \& Bessen, D. E. (2007). Role of streptococcal T antigens in superficial skin infection. J Bacteriol 189, 1426-1434.

Luca-Harari, B., Ekelund, K., van der Linden, M., Staum-Kaltoft, M., Hammerum, A. M. \& Jasir, A. (2008). Clinical and epidemiological aspects of invasive Streptococcus pyogenes infections in Denmark during 2003 and 2004. J Clin Microbiol 46, 79-86.

Manetti, A. G., Zingaretti, C., Falugi, F., Capo, S., Bombaci, M., Bagnoli, F., Gambellini, G., Bensi, G., Mora, M. \& other authors (2007). Streptococcus pyogenes pili promote pharyngeal cell adhesion and biofilm formation. Mol Microbiol 64, 968-983.

Miranda, A. G., Singh, K. V. \& Murray, B. E. (1991). DNA fingerprinting of Enterococcus faecium by pulsed-field gel electrophoresis may be a useful epidemiological tool. J Clin Microbiol 29, 2752-2757.

Mora, M., Bensi, G., Capo, S., Falugi, F., Zingaretti, C., Manetti, A. G., Maggi, T., Taddei, A. R., Grandi, G. \& Telford, J. L. (2005). Group A streptococcus produce pilus-like structures containing protective antigens and Lancefield T antigens. Proc Natl Acad Sci U S A 102, 15641-15646.

Musumeci, R., Bue, C. L., Milazzo, I., Nicoletti, G., Serra, A., Speciale, A. \& Blandino, G. (2003). Internalization-associated proteins among Streptococcus pyogenes isolated from asymptomatic carriers and children with pharyngitis. Clin Infect Dis 37, 173-179.

Nakata, M., Podbielski, A. \& Kreikemeyer, B. (2005). MsmR, a specific positive regulator of the Streptococcus pyogenes FCT pathogenicity region and cytolysin-mediated translocation system genes. Mol Microbiol 57, 786-803.

Nakata, M., Köller, T., Moritz, K., Ribardo, D., Jonas, L., Mclver, K. S., Sumitomo, T., Terao, Y., Kawabata, S. \& other authors (2009). Mode of expression and functional characterization of FCT-3 pilus region encoded proteins in the Streptococcus pyogenes serotype M49. Infect Immun 77, 32-44.

Nordstrand, A., Norgren, M. \& Holm, S. E. (1999). Pathogenic mechanism of acute post-streptococcal glomerulonephritis. Scand J Infect Dis 31, 523-537.

Orscheln, R. C., Johnson, D. R., Olson, S. M., Presti, R. M., Martin, J. M., Kaplan, E. L. \& Storch, G. A. (2005). Intrinsic reduced susceptibility of serotype 6 Streptococcus pyogenes to fluoroquinolone antibiotics. J Infect Dis 191, 1272-1279.

Parsek, M. R. \& Singh, P. K. (2003). Bacterial biofilms: an emerging link to disease pathogenesis. Annu Rev Microbiol 57, 677-701.

Podbielski, A., Melzer, B. \& Lütticken, R. (1991). Application of the polymerase chain reaction to study the $\mathrm{M}$ protein(-like) gene family in $\beta$-hemolytic streptococci. Med Microbiol Immunol 180, 213-227.

Podbielski, A., Krebs, B. \& Kaufhold, A. (1994). Genetic variability of emm-related genes of the large vir regulon of group A streptococci: potential intra- and intergenomic recombinational events. Mol Gen Genet 243, 691-698.
Podbielski, A., Woischnik, M., Leonard, B. A. B. \& Schmidt, K.-H. (1999). Characterization of $n r a$, a global negative regulator gene in group A streptococci. Mol Microbiol 31, 1051-1064.

Potter, E. V., Svartman, M., Mohammed, I., Cox, R., Poon-King, T. \& Earle, D. P. (1978). Tropical acute rheumatic fever and associated streptococcal infections compared with concurrent acute glomerulonephritis. J Pediatr 92, 325-333.

Ramachandran, V., McArthur, J. D., Behm, C. E., Gutzeit, C., Dowton, M., Fagan, P. K., Towers, R., Currie, B., Sriprakash, K. S. \& Walker, M. J. (2004). Two distinct genotypes of prtF2, encoding a fibronectin binding protein, and evolution of the gene family in Streptococcus pyogenes. J Bacteriol 186, 7601-7609.

Reinert, R. R., Lütticken, R., Bryskier, A. \& Al-Lahham, A. (2003). Macrolide-resistant Streptococcus pneumoniae and Streptococcus pyogenes in the pediatric population in Germany during 2000-2001. Antimicrob Agents Chemother 47, 489-493.

Reinert, R. R., Lütticken, R., Sutcliffe, J. A., Tait-Kamradt, A., Cil, M. Y., Schorn, H. M., Bryskier, A. \& Al-Lahham, A. (2004). Clonal relatedness of erythromycin-resistant Streptococcus pyogenes isolates in Germany. Antimicrob Agents Chemother 48, 1369-1373.

Robinson, D. A., Sutcliffe, J. A., Tewodros, W., Manoharan, A. \& Bessen, D. E. (2006). Evolution and global dissemination of macrolide-resistant group A streptococci. Antimicrob Agents Chemother 50, 2903-2911.

Sauermann, R., Gattringer, R., Graninger, W., Buxbaum, A. \& Georgopoulos, A. (2003). Phenotypes of macrolide resistance of group A streptococci isolated from outpatients in Bavaria and susceptibility to 16 antibiotics. J Antimicrob Chemother 51, 53-57.

Spinaci, C., Magi, G., Zampaloni, C., Vitali, L. A., Paoletti, C., Catania, M. R., Prenna, M., Ferrante, L., Ripa, S. \& other authors (2004). Genetic diversity of cell-invasive erythromycin-resistant and -susceptible group A streptococci determined by analysis of the RD2 region of the prtF1 gene. J Clin Microbiol 42, 639-644.

Stevens, D. L. (1996). Invasive group A streptococcal disease. Infect Agents Dis 5, 157-166.

Szczypa, K., Sadowy, E., Izdebski, R. \& Hryniewicz, W. (2004). A rapid increase in macrolide resistance in Streptococcus pyogenes isolated in Poland during 1996-2002. J Antimicrob Chemother 54, 828-831.

Szczypa, K., Sadowy, E., Izdebski, R., Strakova, L. \& Hryniewicz, W. (2006). Group A streptococci from invasive-disease episodes in Poland are remarkably divergent at the molecular level. J Clin Microbiol 44, 3975-3979.

Takemura, N., Noiri, Y., Ehara, A., Kawahara, T., Noguchi, N. \& Ebisu, S. (2004). Single species biofilm-forming ability of root canal isolates on gutta-percha points. Eur J Oral Sci 112, 523-529.

Vlaminckx, B., van Pelt, W., Schouls, L., van Silfhout, A., Elzenaar, C., Mascini, E., Verhoef, J. \& Schellekens, J. (2004). Epidemiological features of invasive and noninvasive group A streptococcal disease in the Netherlands, 1992-1996. Eur J Clin Microbiol Infect Dis 23, 434444.

Wahl, R. U., Lütticken, R., Stanzel, S., van der Linden, M. \& Reinert, R. R. (2007). Epidemiology of invasive Streptococcus pyogenes infections in Germany, 1996-2002: results from a voluntary laboratory surveillance system. Clin Microbiol Infect 13, 1173-1178. 